\title{
Effects of exenatide on cardiac function, perfusion, and energetics in type 2 diabetic patients with cardiomyopathy: a randomized controlled trial against insulin glargine
}

\author{
Weena J. Y. Chen ${ }^{1 *}$, Michaela Diamant ${ }^{1 \wedge}$, Karin de Boer ${ }^{2}$, Hendrik J. Harms ${ }^{3}$, Lourens F. H. J. Robbers ${ }^{2}$,
} Albert C. van Rossum², Mark H. H. Kramer', Adriaan A. Lammertsma ${ }^{3}$ and Paul Knaapen ${ }^{2}$

\begin{abstract}
Background: Multiple bloodglucose-lowering agents have been linked to cardiovascular events. Preliminary studies showed improvement in left ventricular (LV) function during glucagon-like peptide-1 receptor agonist administration. Underlying mechanisms, however, are unclear. The purpose of this study was to investigate myocardial perfusion and oxidative metabolism in type 2 diabetic (T2DM) patients with LV systolic dysfunction as compared to healthy controls. Furthermore, effects of 26-weeks of exenatide versus insulin glargine administration on cardiac function, perfusion and oxidative metabolism in T2DM patients with LV dysfunction were explored.

Methods and results: Twenty-six T2DM patients with LV systolic dysfunction (cardiac magnetic resonance (CMR) derived LV ejection fraction (LVEF) of $47 \pm 13 \%$ ) and 10 controls (LVEF of $59 \pm 4 \%, P<0.01$ as compared to patients) were analyzed. Both myocardial perfusion during adenosine-induced hyperemia $(P<0.01)$, and coronary flow reserve $(\mathrm{P}<0.01)$, measured by $\left[{ }^{15} \mathrm{O}\right] \mathrm{H}_{2} \mathrm{O}$ positron emission tomography $(\mathrm{PET})$, were impaired in T2DM patients as compared to healthy controls. Myocardial oxygen consumption and myocardial efficiency, measured using $\left[{ }^{11} \mathrm{C}\right]$ acetate PET and CMR derived stroke volume, were not different between the groups. Eleven patients in the exenatide group and 12 patients in the insulin glargine group completed the trial. Systemic metabolic control was improved after both treatments, although, no changes in cardiac function, perfusion and metabolism were seen after exenatide or insulin glargine.
\end{abstract}

Conclusions: T2DM patients with LV systolic dysfunction did not have altered myocardial efficiency as compared to healthy controls. Exenatide or insulin glargine had no effects on cardiac function, perfusion or oxidative metabolism. Trial registration NCT00766857

Keywords: Diabetes mellitus type 2, Exenatide, Cardiac function, Myocardial perfusion, Myocardial oxidative metabolism

\section{Background}

Type 2 diabetes mellitus (T2DM) is associated with an increased risk of heart failure (HF), even after adjusting for coronary artery disease (CAD) and hypertension [1].

\footnotetext{
${ }^{*}$ Correspondence: j.chen@vumc.nl

$\wedge$ Deceased

${ }^{1}$ Diabetes Center/Department of Internal Medicine, VU University Medical Center, de Boelelaan 1117, 1081 HV Amsterdam, The Netherlands Full list of author information is available at the end of the article
}

The optimal bloodglucose-lowering therapy in T2DM patients and HF is still under debate. Multiple agents [2-4] have been linked to cardiovascular events. Other agents $[5,6]$ have been associated with a lower risk of cardiovascular events.

Preliminary studies have shown recovery of left ventricular (LV) function during glucagon-like peptide-1 (GLP-1) administration in HF patients irrespective of the diabetic status $[7,8]$. However, underlying mechanisms 
for this effect remain to be elucidated. Small scaled studies suggested enhanced endothelial function and increased perfusion by GLP-1 [9, 10]. Moreover, shift towards augmented glucose metabolism has favorable effects on cardiac energetics [11]. Both phenomena have important prognostic relevance [12, 13]. At present, effects of GLP-1 receptor agonists (RA) on myocardial perfusion and energetics in T2DM patients with LV systolic dysfunction are unclear. In view of the diabetes pandemic and the associated high risk of HF, bloodglucose-lowering agents that can be prescribed safely are of great importance. Therefore, the aim of this study was to examine effects of GLP-1RA on cardiac function, myocardial perfusion, and energetics in T2DM patients with LV systolic dysfunction compared to insulin glargine.

\section{Methods}

\section{Participants}

T2DM patients with LV dysfunction, LV ejection fraction (LVEF) $<50 \%$ [as documented in the medical records, measured using echocardiogram, radionuclide angiogram or cardiovascular magnetic resonance imaging (CMR)], above 18 years, body mass index (BMI) of $25-40 \mathrm{~kg} \mathrm{~m}^{-2}$, hemoglobin A1C (HbA1c) of $6.5-10.0 \%$ (48-86 mmol mol $\left.\mathrm{m}^{-1}\right)$, were randomized, at an allocation ratio of $1: 1$, open-label, to exenatide or insulin glargine on top of ongoing use of oral glucose-lowering agents (metformin or metformin and sulfonylurea) after a run-in period of 10 weeks. Exclusion criteria were renal or liver impairment, malignancy, cardiovascular events $<3$ months, insulin, thiazolidinediones, incretinbased therapies $<4$ months and chronic glucocorticoid use. Patients with contraindication for positron emission tomography (PET) or CMR (e.g. claustrophobia, implanted metal devices, rhythm other than sinus) were excluded. Healthy BMI-matched subjects with normal glucose metabolism (assessed by 75-g oral glucose-tolerance test) served as controls. All participants gave written informed consent. The study protocol was approved by the Medical Ethics Review committee of the VU University Medical Center, and was performed in full compliance with the declaration of Helsinki.

\section{Study procedures}

$5 \mu \mathrm{g}$ exenatide twice daily was injected subcutaneously, $15 \mathrm{~min}$ before breakfast and dinner, for 4 weeks, followed by an increase to $10 \mu \mathrm{g}$ twice daily. Insulin glargine was initiated at $10 \mathrm{IU}$ once daily, injected subcutaneously according to normal standard dosages. Patients were instructed to increase the dose based on fasting blood glucose levels $\left(<5.6 \mathrm{mmol} \mathrm{L}^{-1}\right)$ according to a prespecified treat-to-target algorithm [14]. CMR and PET were performed in the morning within $4 \mathrm{~h}$ after a standardized breakfast and study medication, prior to randomization, and patients underwent follow-up measurements after 26 weeks of treatment.

\section{Outcomes}

The primary outcome in the current study was effects on LVEF after 26-week treatment of exenatide versus insulin glargine in T2DM patients with LV systolic dysfunction. Secondary outcomes were differences in myocardial perfusion and energetics in T2DM patients with LV systolic dysfunction versus healthy BMI-matched healthy controls, and effects on myocardial perfusion and energetics after 26-week treatment of exenatide versus insulin glargine in T2DM patients with LV systolic dysfunction.

\section{CMR}

Measurements were performed using a 1.5 Tesla wholebody magnetic resonance imaging (MRI) scanner (MAGNETOM Avanto, Siemens, Erlangen, Germany). All images were acquired with electrocardiographic triggering, during repeated expiration breath-holds. Cine imaging was used to measure LV dimensions and systolic function. After localizing scout scans, cine images were acquired using a retrospectively triggered, balanced steady-state free precession (SSFP) gradient echo sequence in three long axis views (2-, 3-, and 4-chamber views). Subsequently, short axis images (slice thickness $5 \mathrm{~mm}$, gap $5 \mathrm{~mm}$ ), covering the whole LV from mitral valve annulus to apex, was acquired. Delayed contrast enhancement (DCE) imaging was used to quantify myocardial scarring. DCE images were acquired 15-20 min after intravenous administration of $0.2 \mathrm{mmol} \mathrm{kg}{ }^{-1}$ gadolinium-based contrast agent (gadoterate meglumine, Dotarem ${ }^{\circledR}$, Guerbet, France). DCE images were acquired in the same short and long axis views as those used for cine imaging. For this purpose, a 2D segmented inversion-recovery prepared gradient echocardiography sequence was applied.

\section{CMR data analysis}

LVEF and global LV parameters were quantified using MASS software package (MEDIS, Leiden, The Netherlands). Endocardial and epicardial borders were outlined manually in end-diastolic and end-systolic frames of all short-axis slices. Presence, and degree of fibrosis, of total ventricular mass, were quantified from DCE images using the 5-standard deviation method [15].

\section{PET}

PET assessments were performed using a hybrid PET/ computed tomography (CT) scanner (Gemini TF-64, Philips Healthcare, Best, The Netherlands). Oxidative metabolism was measured using $\left[{ }^{11} \mathrm{C}\right]$ acetate and perfusion using $\left[{ }^{15} \mathrm{O}\right] \mathrm{H}_{2} \mathrm{O}$. 


\section{$\left[{ }^{11}\right.$ C]acetate PET scan}

After a survey scan to position the patient, $370 \mathrm{MBq}$ of $\left[{ }^{11} \mathrm{C}\right]$ acetate was injected intravenously $(5 \mathrm{~mL}$ bolus, infusion speed $\left.0.8 \mathrm{~mL} \mathrm{~s}^{-1}\right)$ followed by a saline flush $(35 \mathrm{~mL}$, infusion speed $2 \mathrm{~mL} \mathrm{~s}^{-1}$ ). Simultaneously, an emission scan was started, acquiring list mode data for $50 \mathrm{~min}$. Using a low-dose respiration-averaged CT scan $(55 \mathrm{~mA}$, rotation time $1.5 \mathrm{~s}$, pitch 0.825 , collimation $64 \times 0.625$, acquiring $20 \mathrm{~cm}$ in $11 \mathrm{~s}$ ) tissue density was measured and $\left[{ }^{11} \mathrm{C}\right]$ acetate scan was corrected for tissue attenuation. Dynamic images were reconstructed using 3D-row action maximum likelihood algorithm into 36 frames $(1 \times 10,8 \times 5,4 \times 10,3 \times 20,5 \times 30,5 \times 60,4 \times 150$, $6 \times 300$ s), applying all appropriate corrections.

\section{$\left[{ }^{15} \mathrm{O}\right] \mathrm{H}_{2} \mathrm{O}$ PET scan}

Hyperemic scan was performed during adenosine intravenously at a rate of $140 \mathrm{mcg} \mathrm{kg} \mathrm{kg}^{-1} \mathrm{~min}^{-1}$. Rest and hyperemic scans were acquired in a list mode of $6 \mathrm{~min}$. At the start of each perfusion scan, $370 \mathrm{MBq}\left[{ }^{15} \mathrm{O}\right] \mathrm{H}_{2} \mathrm{O}$ was injected intravenously ( $5 \mathrm{~mL}$ bolus, infusion speed $0.8 \mathrm{~mL} \mathrm{~s}^{-1}$ ) followed by a saline flush $(35 \mathrm{~mL}$, infusion speed $2 \mathrm{~mL} \mathrm{~s}^{-1}$ ). Both scans were followed by a low-dose respiration-averaged CT scan $(55 \mathrm{~mA}$, rotation time $1.5 \mathrm{~s}$, pitch 0.825 , collimation $64 \times 0.625$, acquiring $20 \mathrm{~cm}$ in $11 \mathrm{~s})$. Images were reconstructed using 3D-row action maximum likelihood algorithm into 22 frames $(1 \times 10$, $8 \times 5,4 \times 10,2 \times 15,3 \times 20,2 \times 30$, and $2 \times 60$ s), with application of all appropriate corrections. BP was measured every 3 min during the entire scan. An interval of at least 10 min was used between the end of the first and the start of the second $\left[{ }^{15} \mathrm{O}\right] \mathrm{H}_{2} \mathrm{O}$ scan to allow for decay of radioactivity to background levels.

\section{PET data analysis}

Regions of interest were drawn on resliced short axis projections of a static $\left[{ }^{11} \mathrm{C}\right]$ acetate image during maximum myocardial uptake according to the standardized AHA 17-segment model [16]. To obtain arterial input function, additional regions were drawn within the ascending aorta in at least five consecutive planes. Subsequently, all regions were projected onto all frames of the dynamic $\left[{ }^{11} \mathrm{C}\right]$ acetate scan to generate time-activity curves (TAC). A single-tissue compartment model with model based corrections for spillover, partial volume and recirculating $\left[{ }^{11} \mathrm{C}\right]$ activity was used to derive $\mathrm{k}_{2}$, representing the rate constant for tracer washout from myocardial tissue, described previously $[17,18]$. Quantitative parametric myocardial blood flow (MBF) images were generated using software developed in-house [19]. MBF was expressed in $\mathrm{mL} \mathrm{min}^{-1} \mathrm{~g}^{-1}$ of perfusable tissue.

\section{Data analysis}

Rate pressure product (RPP) was obtained by multiplying systolic BP (SBP) with heart rate (HR). Resting MBF corrected for RPP was derived as MBF at rest divided by RPP at rest, multiplied by $10^{4}$. Coronary flow reserve (CFR) was defined as the ratio between hyperemic and resting MBF and CFR corrected for RPP as the ratio between hyperemic and resting MBF corrected for RPP. External work was defined as the product of mean arterial pressure and CMR derived stroke volume, converted to units of energy (Joule). The caloric equivalent of $1 \mathrm{~mL}$ oxygen $\approx 20$ Joule, whereas $1 \mathrm{~mm} \mathrm{Hg} \mathrm{mL}$ equals $1.33 \cdot 10^{-4}$ Joule [20]. Myocardial oxygen consumption $\left(\mathrm{MVO}_{2}\right)$ in $\mathrm{mL} \mathrm{g}^{-1} \mathrm{~min}^{-1}$ was derived from $\mathrm{k}_{2}$ using estimated $\mathrm{MVO}_{2}=1.35 \cdot \mathrm{k}_{2}-0.0096$ as described previously [21]. Myocardial efficiency was calculated as (External work $\cdot \mathrm{HR}) /\left(\mathrm{MVO}_{2} \cdot \mathrm{LV}\right.$ mass.20) [22].

\section{Statistical analysis}

Data are expressed as means \pm standard deviations (for normally distributed data) or medians (interquartile range). $T$ test or Mann-Whitney U-test was used to determine within-group differences. Between-group comparisons were performed using linear regression analysis with adjustments for intervention group and baseline values. Correlation coefficients were calculated using Pearson's correlation. Statistical analyses were performed using SPSS software version 20.0 (IBM corporation, New York, US). P-value $<0.05$ was considered as statistically significant. Sample size calculations are based on the primary efficacy endpoint, i.e. LVEF. According to previous studies using GLP- 1 and based on clinical experience, an absolute LVEF improvement of $5 \%$ is regarded as attainable and clinically relevant. In order to detect an absolute 5\% improvement of LVEF from baseline in patients treated with exenatide, relative to those treated by the comparator insulin glargine (SD absolute $4 \% ; \alpha=0.05, \beta=0.10), 13$ patients in each group will be needed.

\section{Results}

\section{T2DM patients versus controls}

Twenty-seven T2DM male patients (Fig. 1) and 10 male controls were included. One patient was excluded from analysis, because of the necessity of cardioverter defibrillation therapy (Fig. 1). Baseline characteristics are listed in Table 1. T2DM patients were older than controls and well matched for BMI. Median diabetes duration was 8 years. Twenty-four of 26 T2DM patients had known CAD. Waist circumference was greater in T2DM patients than in controls. Furthermore, T2DM patients had impaired metabolic control, with higher HbA1c, 


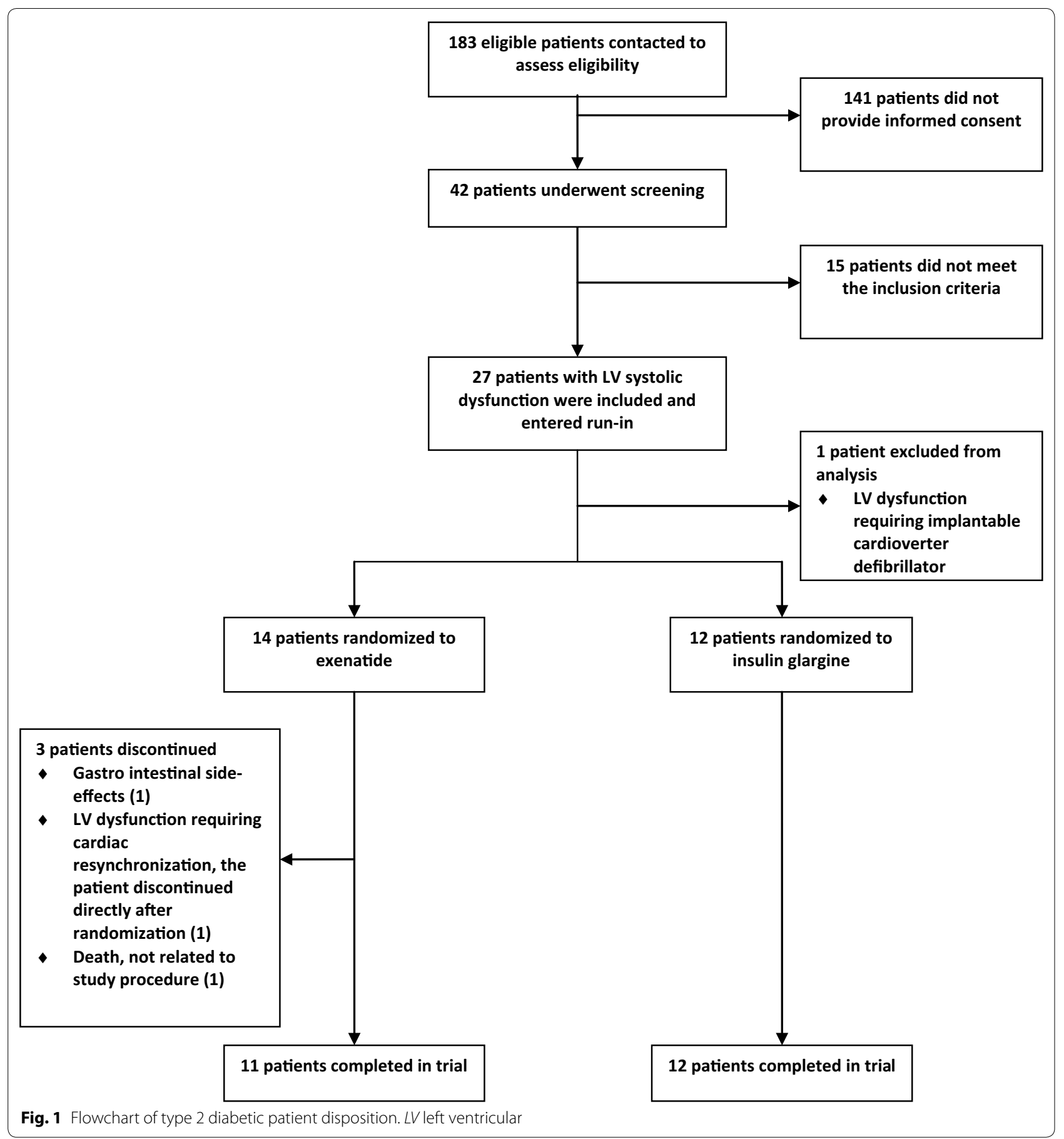

triglycerides-and non-esterified fatty acid levels, and lower high-density lipoprotein (HDL) cholesterol levels. Total cholesterol and low-density lipoprotein (LDL) cholesterol levels did not differ between the groups. No difference was seen in estimated glomerular filtration rate. Yet, albumin-to-creatinine ratio was higher in T2DM patients.
Hemodynamic parameters during perfusion scans are shown in Table 2. At rest, HR, SBP, and RPP were higher in T2DM patients. Hyperemia induced increased HR and RPP in both groups. In controls, hyperemia caused increased SBP and mean arterial pressure. Diastolic BP (DBP) was unaffected during hyperemia, although DBP was higher in controls than in T2DM patients during 
Table 1 Baseline characteristics healthy controls and type 2 diabetic patients

\begin{tabular}{|c|c|c|c|}
\hline & Controls $(n=10)$ & T2DM patients $(n=26)$ & $P$ \\
\hline Age, years & $59 \pm 5$ & $66 \pm 5$ & $<0.01$ \\
\hline Diabetes duration, years & NA & $8(5-11)$ & NA \\
\hline Coronary artery disease, $\mathrm{n}(\%)$ & NA & $24(92 \%)$ & NA \\
\hline Male, n (\%) & $10(100)$ & $26(100)$ & 1.00 \\
\hline $\mathrm{BMI}, \mathrm{kg} \mathrm{m}^{-2}$ & $29.4 \pm 2.2$ & $29.8 \pm 3.1$ & 0.74 \\
\hline Waist, $\mathrm{cm}$ & $103 \pm 6$ & $110 \pm 10$ & 0.04 \\
\hline \multicolumn{4}{|l|}{ Medication } \\
\hline ACE inhibitor, n (\%) & NA & $15(56 \%)$ & NA \\
\hline Angiotensin II blocker, n (\%) & NA & $10(37 \%)$ & NA \\
\hline Beta blocker, n (\%) & NA & $23(85 \%)$ & NA \\
\hline Statins, n (\%) & NA & $24(89 \%)$ & NA \\
\hline \multicolumn{4}{|c|}{ Biochemical measurements (in fasten state) } \\
\hline $\mathrm{HbA} 1 \mathrm{c}, \%$ & $5.5 \pm 0.2$ & $7.5 \pm 1.3$ & $<0.01$ \\
\hline Plasma glucose, $\mathrm{mmol} \mathrm{L}^{-1}$ & $5.5 \pm 0.3$ & $9.7 \pm 2.6$ & $<0.01$ \\
\hline Total cholesterol, mmol $\mathrm{L}^{-1}$ & $5.7 \pm 0.7$ & $4.3 \pm 1.0$ & $<0.01$ \\
\hline $\mathrm{HDL}$ cholesterol, mmol $\mathrm{L}^{-1}$ & $1.4(1.2-1.5)$ & $1.0(0.9-1.2)$ & $<0.01$ \\
\hline LDL cholesterol, mmol L & $3.9 \pm 0.7$ & $2.3 \pm 0.9$ & $<0.01$ \\
\hline Triglycerides, mmol $\mathrm{L}^{-1}$ & $1.1(0.9-1.2)$ & $1.6(1.1-2.1)$ & 0.02 \\
\hline Non-esterified fatty acids, $\mathrm{mmol} \mathrm{L}^{-1}$ & $0.4 \pm 0.1$ & $0.5 \pm 0.2$ & 0.03 \\
\hline eGFR (MDRD), mL min ${ }^{-1} 1.73 \mathrm{~m}^{-2}$ & $89 \pm 14$ & $86 \pm 28$ & 0.80 \\
\hline Albumin-to-creatinin ratio, $\mathrm{g} \mathrm{mol}^{-1}$ & $0.4(0.3-0.5)$ & $1.3(0.6-2.4)$ & $<0.01$ \\
\hline
\end{tabular}

Data are mean $\pm S D$, median (interquartile range) or numbers of patients (percentage)

T2DM type 2 diabetes mellitus, NA not applicable, BMI body mass index, ACE angiotensin converting enzyme, HbA1c hemoglobin A1c, HDL high-density lipoprotein, $L D L$ low-density lipoprotein, eGFR estimated glomerular filtration rate, MDRD modification of diet in renal disease

hyperemia. No differences in HR, SBP, or RPP during hyperemia between groups were observed.

In Table 3 cardiac parameters are summarized. LV volumes and mass were not different between groups. LVEF, resting and hyperemic MBF, and CFR were impaired in T2DM patients compared to controls. T2DM patients had $6.3 \mathrm{~g}(0-11.3 \mathrm{~g})$ of LV fibrosis as measured bij DCE, while controls did not show any. After correcting for RPP, differences in resting MBF and CFR were no longer present (Table 3; Fig. 2). $\mathrm{MVO}_{2}$ and myocardial efficiency were not different between the groups. Myocardial efficiency was related with LVEF in controls and T2DM (Fig. 3).

Effects of exenatide and insulin glargine in T2DM patients Eleven patients on exenatide and 12 patients on insulin glargine completed the trial (Fig. 1). Three of 14 patients randomized to exenatide discontinued after randomization, because of gastrointestinal side effects, severe LV dysfunction requiring cardiac resynchronization therapy, and one patient died from endocarditis. At baseline, the groups were well matched (Tables 4,5 ). Compared to insulin glargine, exenatide reduced weight, indicated by a reduced BMI, and waist circumference (Table 4). After
26 weeks of both treatments, HbA1c decreased without between-group differences (Table 4). At follow-up, only patients on insulin glargine had decreased fasting plasma glucose (Table 4). Total cholesterol and triglycerides levels decreased after 26 weeks of exenatide, but betweengroup analyses showed no differences. Both HDL and LDL cholesterol, as well as non-esterified fatty acid levels were unchanged in both groups at follow-up (Table 4). Renal function, depicted in estimated glomerular filtration rate and albumin-to-creatinine ratio, was unaffected after both treatments (Table 4).

At follow-up, HR was increased in both groups. SBP and DBP, and mean arterial pressure, at rest and during hyperemia, were unaffected after both treatments. At follow-up, although resting RPP was increased in the exenatide group $(\mathrm{P}=0.02)$, between-group analysis did not show differences for any hemodynamic parameters (not shown).

LV volumes and mass did not change after both treatments (Table 5). Neither LVEF, nor total DCE area, were altered at follow-up (Table 5; Fig. 4). No differences in resting (Table 5) or hyperemic MBF, as well as CFR (Table 5; Fig. 5a, c), were seen after exenatide or insulin glargine. However, RPP corrected resting MBF was 
Table 2 Hemodynamic parameters at rest and during hyperemia in healthy controls and type 2 diabetic patients

\begin{tabular}{|c|c|c|c|}
\hline & Controls $(n=10)$ & T2DM patients $(n=26)$ & $P$ \\
\hline \multicolumn{4}{|c|}{ Heart rate, beats $\min ^{-1}$} \\
\hline Rest & $55 \pm 3$ & $64 \pm 11$ & $<0.01$ \\
\hline Hyperemia & $76 \pm 10$ & $79 \pm 13$ & 0.42 \\
\hline P-value & $<0.01$ & $<0.01$ & \\
\hline \multicolumn{4}{|c|}{ Systolic blood pressure, $\mathrm{mmHg}$} \\
\hline Rest & $116 \pm 6$ & $127 \pm 17$ & 0.01 \\
\hline Hyperemia & $129 \pm 11$ & $131 \pm 19$ & 0.68 \\
\hline P-value & $<0.01$ & 0.12 & \\
\hline \multicolumn{4}{|c|}{ Diastolic blood pressure, $\mathrm{mmHg}$} \\
\hline Rest & $71 \pm 6$ & $66 \pm 10$ & 0.26 \\
\hline Hyperemia & $74 \pm 6$ & $67 \pm 8$ & 0.02 \\
\hline P-value & 0.21 & 0.47 & \\
\hline \multicolumn{4}{|c|}{ Mean arterial pressure, $\mathrm{mmHg}$} \\
\hline Rest & $86 \pm 5$ & $87 \pm 12$ & 0.72 \\
\hline Hyperemia & $92 \pm 5$ & $89 \pm 11$ & 0.17 \\
\hline P-value & 0.02 & 0.23 & \\
\hline \multicolumn{4}{|c|}{ Rate pressure product, $\mathrm{mm} \mathrm{Hg} \mathrm{min}^{-1}$} \\
\hline Rest & $6318 \pm 515$ & $8050 \pm 1430$ & $<0.01$ \\
\hline Hyperemia & $9673 \pm 1123$ & $10,355 \pm 2217$ & 0.23 \\
\hline P-value & $<0.01$ & $<0.01$ & \\
\hline
\end{tabular}

Data are mean $\pm S D$

T2DM type 2 diabetes mellitus

decreased after exenatide, although between-group analysis did not show changes (Table 5; Fig. 5a, b). $\mathrm{MVO}_{2}$, and myocardial efficiency, were unchanged after both treatments (Table 5; Fig. 6).

\section{Discussion}

The purpose of this study was to investigate the effects of exenatide compared to insulin glargine on myocardial function, perfusion, and energetics in T2DM patients with systolic dysfunction, using a BMI-matched controls as reference. As expected, T2DM patients had impaired metabolic control, with higher HbA1c, as well as fasting glucose, and non-esterified fatty acid levels, compared to controls. In addition, T2DM was associated with coronary vasomotor dysfunction, whereas myocardial energetics did not differ between groups. Systemic metabolic control improved to the same degree after 26 weeks of exenatide and insulin glargine. Finally, significant weight loss was seen after exenatide. Nevertheless, myocardial function, perfusion, and energetics were unaffected by both therapies.

\section{Myocardial perfusion and energetics in T2DM patients versus controls}

Resting MBF was increased in T2DM as compared with controls. After correcting for hemodynamics, however, these differences were no longer apparent. Cardiovascular autonomic dysfunction is common in T2DM [23], resulting in elevated resting HR. Furthermore, as central obesity and insulin resistance are more prevalent, T2DM is associated with hypertension [24]. These changes will lead to augmentation of myocardial metabolic demand and, consequently, affect resting MBF. Existing PET data on resting MBF in T2DM are inconsistent. Without correction for hemodynamics, resting MBF in T2DM has been described as comparable to controls [25-28]. RPPs

Table 3 Imaging parameters in healthy controls and type 2 diabetic patients

\begin{tabular}{|c|c|c|c|}
\hline & Controls $(n=10)$ & T2DM patients $(n=26)$ & $\mathbf{P}$ \\
\hline \multicolumn{4}{|l|}{ CMR } \\
\hline LVEDV, mL & $202 \pm 36$ & $209 \pm 70$ & 0.79 \\
\hline LVESV, mL & $83 \pm 18$ & $117 \pm 75$ & 0.19 \\
\hline LV mass, $g$ & $123 \pm 25$ & $116 \pm 27$ & 0.51 \\
\hline LVEF, \% & $59 \pm 4$ & $47 \pm 13$ & $<0.01$ \\
\hline DCE, g & 0 & $6.3(0-11.3)$ & $<0.01$ \\
\hline \multicolumn{4}{|l|}{ PET } \\
\hline $\mathrm{MBF}$, rest, $\mathrm{mL}^{-1} \mathrm{~min}^{-1} \mathrm{~g}^{-1}$ & $0.75 \pm 0.07$ & $0.86 \pm 0.19$ & 0.02 \\
\hline$M B F$, rest, corrected for RPP, $\mathrm{mL} \mathrm{min}{ }^{-1} \mathrm{~g}^{-1}$ & $1.22 \pm 0.1$ & $1.11 \pm 0.4$ & 0.20 \\
\hline$M B F$, stress, $m L \min ^{-1} g^{-1}$ & $2.64 \pm 0.28$ & $2.05 \pm 0.65$ & $<0.01$ \\
\hline CFR & $3.54 \pm 0.54$ & $2.43 \pm 0.79$ & $<0.01$ \\
\hline CFR, corrected for RPP, $\mathrm{mmHg} \mathrm{min}^{-1}$ & $2.20 \pm 0.35$ & $1.97 \pm 0.74$ & 0.56 \\
\hline External work, Joule & $1.26 \pm 0.26$ & $1.00 \pm 0.31$ & 0.04 \\
\hline $\mathrm{MVO}_{2}, \mathrm{~mL} \mathrm{~g}^{-1} \mathrm{~min}^{-1}$ & $0.09 \pm 0.01$ & $0.08 \pm 0.01$ & 0.23 \\
\hline Myocardial efficiency, \% & $32 \pm 6$ & $35 \pm 9$ & 0.39 \\
\hline
\end{tabular}

Data are mean \pm SD or median (interquartile range)

T2DM type 2 diabetes mellitus, CMR cardiac magnetic resonance, LVEDV left ventricular enddiastolic volume, LVESV left ventricular endsystolic volume, LVEF left ventricular ejection fraction, DCE delayed contrast enhancement, $P E T$ positron emission tomography, MBF myocardial blood flow, RPP rate pressure product, CFR coronary flow reserve, $\mathrm{MVO}_{2}$ myocardial oxygen consumption 


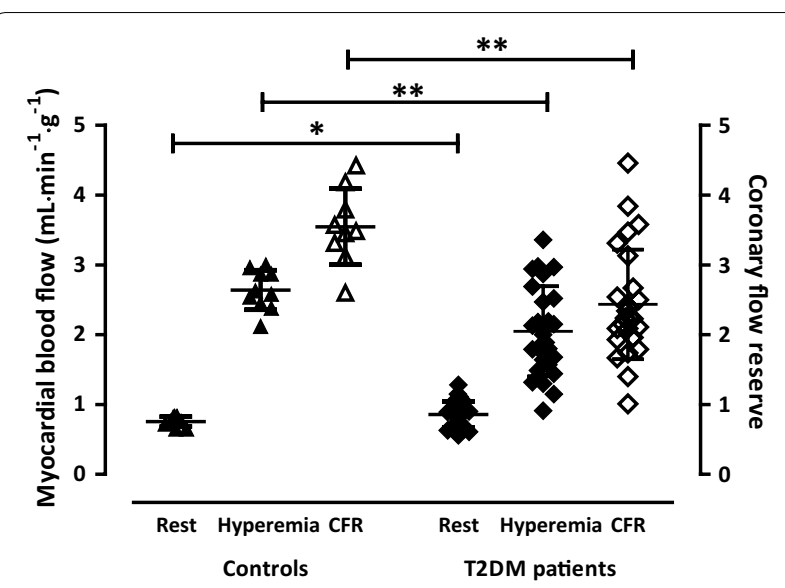

Fig. 2 Resting, and hyperemic myocardial blood flow, and coronary flow reserve (CFR) in healthy controls (triangles) and type 2 diabetic (T2DM) patients (lozenges). ${ }^{*} \mathrm{P}=0.02,{ }^{\dagger} \mathrm{P}<0.01$

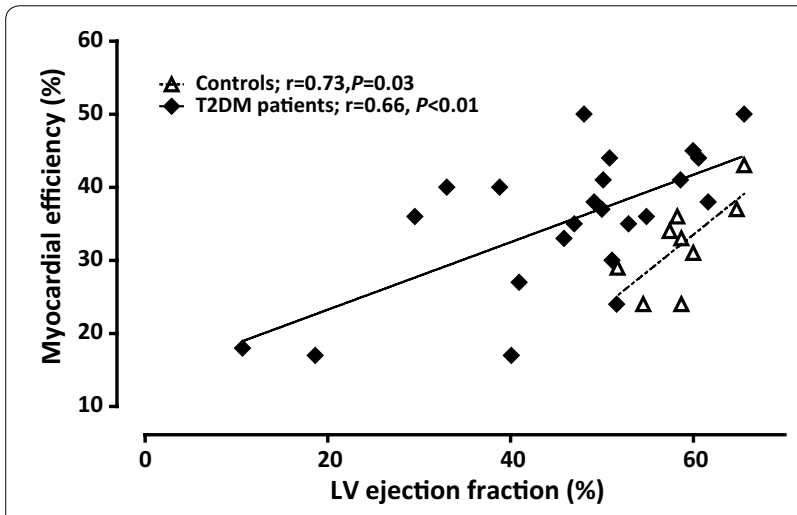

Fig. 3 Association between left ventricular (LV) ejection fraction and myocardial efficiency in healthy controls (white triangles) and type 2 diabetic (T2DM) patients (black lozenges)

in these studies were either increased [26, 28], or comparable between T2DM patients and controls [27]. Not surprisingly, hyperemic MBF was decreased in T2DM patients, which almost all had CAD, compared to controls. However, in T2DM patients without CAD, impaired hyperemic MBF is a common finding as well [29]. As already mentioned, cardiovascular autonomic neuropathy could play a role in impairing vasodilator reserve $[23$, 30]. In T1DM and T2DM patients, cardiac autonomic neuropathy, assessed using $\left[{ }^{11} \mathrm{C}\right]$ hydroxyephedrine imaging, was associated with impaired vasodilator response, resulting in impaired hyperemic MBF as compared to diabetic patients without cardiac autonomic dysfunction [25].

Although all patients had LV systolic dysfunction, $\mathrm{MVO}_{2}$ and myocardial efficiency were comparable to controls. Myocardium relies predominantly on glucose and fatty acids for substrate metabolism [31]. In both T2DM [32] and HF [33], myocardial insulin resistance will lead to a shift in substrate metabolism, resulting in increased fatty acid oxidation, whilst glucose metabolism decreases. Shifting towards fatty acid metabolism is related to an (maximum) increase of $11 \%$ in oxygen consumption to generate the same amount of ATP as for glucose metabolism, resulting in impaired mechanical efficiency [34]. Further deterioration of cardiac function could be the consequence $[11,20]$. There are only a few, conflicting, reports on the use of $\left[{ }^{11} \mathrm{C}\right]$ acetate PET to (indirectly) measure myocardial oxidative metabolism in T2DM patients. In T2DM patients without CAD, diminished myocardial efficiency and efficiency reserve were observed [35]. Peterson et al. [28] found no differences in $\mathrm{MVO}_{2}$ between T2DM patients and BMI-matched controls, although increased fatty acid oxidation was seen in T2DM. Rijzewijk et al. [26] reported increased fatty acid metabolism and decreased myocardial glucose uptake in patients with uncomplicated T2DM compared to healthy controls. Cardiac substrate metabolism was unrelated to LV function. In this study, all patients had LV systolic dysfunction. Idiopathic dilated cardiomyopathy is characterized by decreased myocardial efficiency and LVEF is inversely associated with myocardial fatty acid uptake [36]. Next, in patients with a history of myocardial infarction, myocardial oxygen consumption in residual viable tissues was not changed compared to CAD patients without history of myocardial infarction and controls [37]. However LV work was reduced, resulting in reduced myocardial efficiency [37]. These data are in contrast with the present findings, which did not show altered myocardial energetics in T2DM patients with predominantly ischemic cardiomyopathy.

\section{Effects of exenatide on myocardial function, perfusion, and energetics in T2DM patients}

Hemodynamic parameters did not change after treatment with exenatide or insulin glargine. The majority of trials on GLP-1RA reported decreased SBP [38-40], LDL-cholesterol, and triglycerides [39], as well as improvements in cardiovascular risk markers as HDLcholesterol and hsCRP [40]. These improvements sustained after 5-year of follow-up [41]. Although, increased HR was observed after 6 and 48 h of GLP- 1 infusion in HF patients (with and without T2DM) [42, 43] and in a meta-analysis of 32 trials comparing GLP-1RA with placebo or active comparators, although mean HR increase was less than $2 \mathrm{bpm} \mathrm{[38].}$

To the best of our knowledge, this is one of the first studies in which cardiac effects of long-term GLP-1RA treatment have been investigated in T2DM patients with systolic dysfunction. Neither exenatide nor insulin 
Table 4 26-week treatment of exenatide versus insulin glargine, anthropometric-and biochemical parameters

\begin{tabular}{|c|c|c|c|c|c|c|c|c|}
\hline & \multicolumn{3}{|c|}{ Exenatide $(n=11)$} & \multicolumn{3}{|c|}{ Insulin glargine $(n=12)$} & \multicolumn{2}{|c|}{$\mathrm{P}$ between groups } \\
\hline & Baseline & Follow-up & $\mathbf{P}$ & Baseline & Follow-up & $\mathbf{P}$ & Baseline & Follow-up \\
\hline $\mathrm{BMI}, \mathrm{kg} \mathrm{m}^{-2}$ & $29.0 \pm 2.6$ & $27.6 \pm 3.1$ & $<0.01$ & $29.9 \pm 3.3$ & $30.0 \pm 3.6$ & 0.80 & 0.47 & $<0.01$ \\
\hline Waist, $\mathrm{cm}$ & $108 \pm 9$ & $104 \pm 10.3$ & $<0.01$ & $109 \pm 10$ & $110 \pm 10$ & 0.53 & 0.79 & $<0.01$ \\
\hline \multicolumn{9}{|c|}{ Biochemical measurements (in fasten state) } \\
\hline $\mathrm{HbA} 1 \mathrm{c}, \%$ & $7.7 \pm 1.7$ & $7.1 \pm 1.9$ & $<0.01$ & $7.5 \pm 0.8$ & $6.8 \pm 0.7$ & $<0.01$ & 0.68 & 0.49 \\
\hline Plasma glucose, $\mathrm{mmol} \mathrm{L}^{-1}$ & $10.4 \pm 3.7$ & $9.5 \pm 4.0$ & 0.14 & $9.3 \pm 1.6$ & $7.1 \pm 1.2$ & $<0.01$ & 0.36 & 0.03 \\
\hline Total cholesterol, mmol L ${ }^{-1}$ & $4.1 \pm 1.2$ & $3.8 \pm 0.9$ & 0.04 & $4.3 \pm 0.9$ & $4.1 \pm 0.9$ & 0.06 & 0.74 & 0.25 \\
\hline $\mathrm{HDL}$ cholesterol, mmol $\mathrm{L}^{-1}$ & $0.9(0.7-1.2)$ & $0.9(0.8-1.2)$ & 0.92 & $1.0(0.9-1.1)$ & $1.0(1.0-1.1)$ & 0.29 & 0.54 & 0.24 \\
\hline LDL cholesterol, mmol L-1 & $1.9 \pm 1.1$ & $1.8 \pm 0.9$ & 0.22 & $2.5 \pm 0.7$ & $2.4 \pm 0.8$ & 0.17 & 0.17 & 0.65 \\
\hline Triglycerides, mmol $\mathrm{L}^{-1}$ & $1.8(1.0-3.0)$ & $1.2(0.9-1.7)$ & 0.04 & $1.8(1.1-2.1)$ & $1.4(0.9-1.6)$ & 0.11 & 0.87 & 0.33 \\
\hline Non-esterified fatty acids, $\mathrm{mmol}^{-1}$ & $0.6 \pm 0.1$ & $0.5 \pm 0.2$ & 0.27 & $0.5 \pm 0.1$ & $0.4 \pm 0.1$ & 0.09 & 0.09 & 0.48 \\
\hline eGRF (MDRD), mL $\min ^{-1} 1.73 \mathrm{~m}^{-2}$ & $90 \pm 35$ & $89 \pm 26$ & 0.65 & $82 \pm 22$ & $86 \pm 26$ & 0.29 & 0.52 & 0.37 \\
\hline Albumin-to-creatinin ratio, $\mathrm{g} \mathrm{mol}^{-1}$ & $0.7(0.4-1.3)$ & $0.6(0.4-0.7)$ & 0.88 & $1.5(0.7-3.3)$ & $1.2(0.7-3.5)$ & 0.14 & 0.09 & 0.31 \\
\hline
\end{tabular}

Data are mean $\pm S D$, median (interquartile range) or numbers of patients (percentage)

$B M I$ body mass index, $H b A 1 c$ hemoglobin A1c, HDL high-density lipoprotein, $L D L$ low-density lipoprotein, eGFR estimated glomerular filtration rate, MDRD modification of diet in renal disease

Table 5 Effects of 26-week treatment of exenatide versus insulin glargine on imaging parameters

\begin{tabular}{|c|c|c|c|c|c|c|c|c|}
\hline & \multicolumn{3}{|c|}{ Exenatide $(n=11)$} & \multicolumn{3}{|c|}{ Insulin glargine $(n=12)$} & \multicolumn{2}{|c|}{$\mathrm{P}$ between groups } \\
\hline & Baseline & Follow-up & $\mathbf{P}$ & Baseline & Follow-up & $\mathbf{P}$ & Baseline & Follow-up \\
\hline \multicolumn{9}{|l|}{ CMR } \\
\hline LVEDV, mL & $176 \pm 42$ & $175 \pm 31$ & 0.90 & $206 \pm 38$ & $206 \pm 44$ & 0.93 & 0.10 & 0.49 \\
\hline LVESV, mL & $86 \pm 26$ & $86 \pm 25$ & 1.00 & $104 \pm 29$ & $109 \pm 31$ & 0.11 & 0.13 & 0.25 \\
\hline LV mass, $g$ & $110 \pm 29$ & $105 \pm 20$ & 0.31 & $113 \pm 23$ & $118 \pm 23$ & 0.27 & 0.82 & 0.05 \\
\hline LVEF, \% & $51 \pm 7$ & $52 \pm 7$ & 0.85 & $50 \pm 9$ & $47 \pm 10$ & 0.06 & 0.58 & 0.11 \\
\hline DCE, g & $9.5(1.0-11.4)$ & $8.8(2.2-14.0)$ & 0.74 & $10.5(5.8-21.7)$ & $14.1(5.3-27.4)$ & 0.07 & 0.33 & 0.17 \\
\hline \multicolumn{9}{|l|}{ PET } \\
\hline$M B F$, rest, $\mathrm{mL} \min ^{-1} \mathrm{~g}^{-1}$ & $0.92 \pm 0.18$ & $0.86 \pm 0.11$ & 0.33 & $0.80 \pm 0.18$ & $0.86 \pm 0.17$ & 0.31 & 0.15 & 0.57 \\
\hline MBF, rest, corrected for RPP, $\mathrm{mL} \mathrm{min}^{-1} \mathrm{~g}^{-1}$ & $1.25 \pm 0.46$ & $1.03 \pm 0.26$ & 0.04 & $1.02 \pm 0.26$ & $0.98 \pm 0.16$ & 0.59 & 0.16 & 0.45 \\
\hline MBF, stress, $\mathrm{mL} \min ^{-1} \mathrm{~g}^{-1}$ & $2.29 \pm 0.53$ & $2.20 \pm 0.59$ & 0.57 & $2.00 \pm 0.68$ & $1.94 \pm 0.62$ & 0.46 & 0.28 & 0.79 \\
\hline CFR & $2.59 \pm 0.82$ & $2.60 \pm 0.89$ & 0.96 & $2.51 \pm 0.72$ & $2.24 \pm 0.59$ & 0.20 & 0.82 & 0.22 \\
\hline CFR, corrected for RPP, $\mathrm{mmHg} \mathrm{min}^{-1}$ & $2.03 \pm 0.76$ & $2.26 \pm 0.79$ & 0.19 & $2.04 \pm 0.77$ & $1.98 \pm 0.62$ & 0.65 & 0.96 & 0.16 \\
\hline External work, Joule & $1.06 \pm 0.38$ & $0.98 \pm 0.20$ & 0.35 & $1.12 \pm 0.34$ & $1.12 \pm 0.34$ & 0.99 & 0.70 & 0.21 \\
\hline $\mathrm{MVO}_{2}, \mathrm{~mL} \mathrm{~g}^{-1} \mathrm{~min}^{-1}$ & $0.08 \pm 0.01$ & $0.08 \pm 0.01$ & 0.52 & $0.08 \pm 0.01$ & $0.07 \pm 0.02$ & 0.23 & 0.28 & 0.48 \\
\hline Myocardial efficiency, \% & $36 \pm 8$ & $38 \pm 6$ & 0.53 & $39 \pm 8$ & $40 \pm 11$ & 0.59 & 0.45 & 0.84 \\
\hline
\end{tabular}

Data are mean \pm SD or median (interquartile range). CMR cardiac magnetic resonance

LVEDV left ventricular enddiastolic volume, LVESV left ventricular endsystolic volume, $L V E F$ left ventricular ejection fraction, $D C E$ delayed contrast enhancement, $P E T$ positron emission tomography, MBF myocardial blood flow, RPP rate pressure product, CFR coronary flow reserve, $M V O 2$ myocardial oxygen consumption

glargine had effects on LV dimensions or function. In contrast, initial studies investigating effects of GLP-1 in HF patients showed beneficial effects on LV function [7, 8]. In addition, 5 weeks of GLP-1 infusion improved performance in HF patients, illustrated by increased maximal $\mathrm{O}_{2}$-uptake and 6-min walking distance [7]. Besides, the LEADER trial [6] showed lower incidence of major cardiovascular events and cardiovascular mortality in
T2DM patients with high cardiovascular risk in patients treated with liraglutide during a median follow up of 3.8 years as compared to placebo. In line with the present results, Halbirk et al. [43] could not detect an impact of GLP-1 in 20 patients without diabetes and severe systolic HF who received $48 \mathrm{~h}$ GLP-1 infusion. Furthermore, intervention with liraglutide during 24 weeks did not changed LV systolic function as compared with 


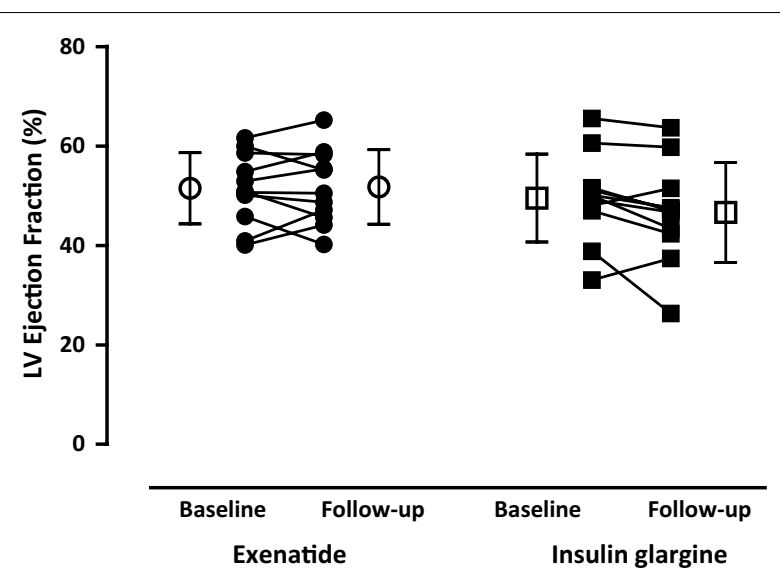

Fig. 4 Left ventricular (LV) ejection fraction in type 2 diabetic patients at baseline and after 26-weeks of exenatide [dots; open dots (mean \pm SD)] versus insulin glargine [squares; open squares (mean $\pm \mathrm{SD})]$

placebo in patients with chronic heart failure with and without T2DM [44]. Moreover, liraglutide administered for 180 days, did not lead to decreased numbers of rehospitalizations, and mortality, as well as N-terminal pro-B-type natriuretic peptide levels in patients recently hospitalized with heart failure and reduced LVEF [45].

Twenty-six weeks of exenatide did not affect resting or hyperemic MBF. After correction for RPP, however, resting MBF was decreased at follow-up. Clinical studies examining effects of GLP-1(RA) on coronary vascular function are scarce. In 8 T2DM patients without cardiovascular disease, resting MBF, measured using $\left[{ }^{13} \mathrm{~N}\right]$ $\mathrm{NH}_{3}$ PET, increased after exenatide during a pancreaticpituitary clamp as compared to placebo [46]. However,
10 weeks of GLP-1RA treatment caused a borderline significant increase in CFR of the left anterior descending artery in T2DM patients without CAD [47]. Others showed increased flow mediated vasodilation after either GLP-1 infusion [9] or 26 weeks of exenatide treatment [48] in T2DM. Furthermore, exenatide inhibited postprandial peripheral vascular endothelial dysfunction after a meal tolerance test, suggesting that it could have a multiphasic anti-atherogenic action involving both glucose and lipid metabolism [49]. Together, these studies and the present data suggest that GLP-1RA has little to no effect on coronary vasomotor function, and small changes in flow may actually be related to hemodynamic alterations. Moreover, most patients in this study had only modest LV dysfunction. This could have contributed to the small differences seen in cardiac function, perfusion and metabolism.

Enhancing myocardial glucose metabolism, thereby improving myocardial efficiency, has been proposed as a therapeutic strategy in HF [12]. However, in the present study $\mathrm{MVO}_{2}$ and myocardial efficiency were unaffected by exenatide. Reports on chronic administration of GLP-1 infusion in dogs [50] with dilated cardiomyopathy, showed increased myocardial glucose uptake. In healthy men, GLP-1 infusion did not affect myocardial glucose uptake [51]. Comparable results with exenatide infusion were shown in T2DM patients without CAD [46]. Furthermore, pre-treatment with GLP-1 in patients with coronary artery disease undergoing elective percutaneous coronary intervention, protected the heart against ischemic LV dysfunction, independent of cardiac substrate use [52]. No changes were seen in the transmyocardial glucose concentration gradients between patients randomized to either GLP-1 or placebo [52]. No changes in myocardial glucose

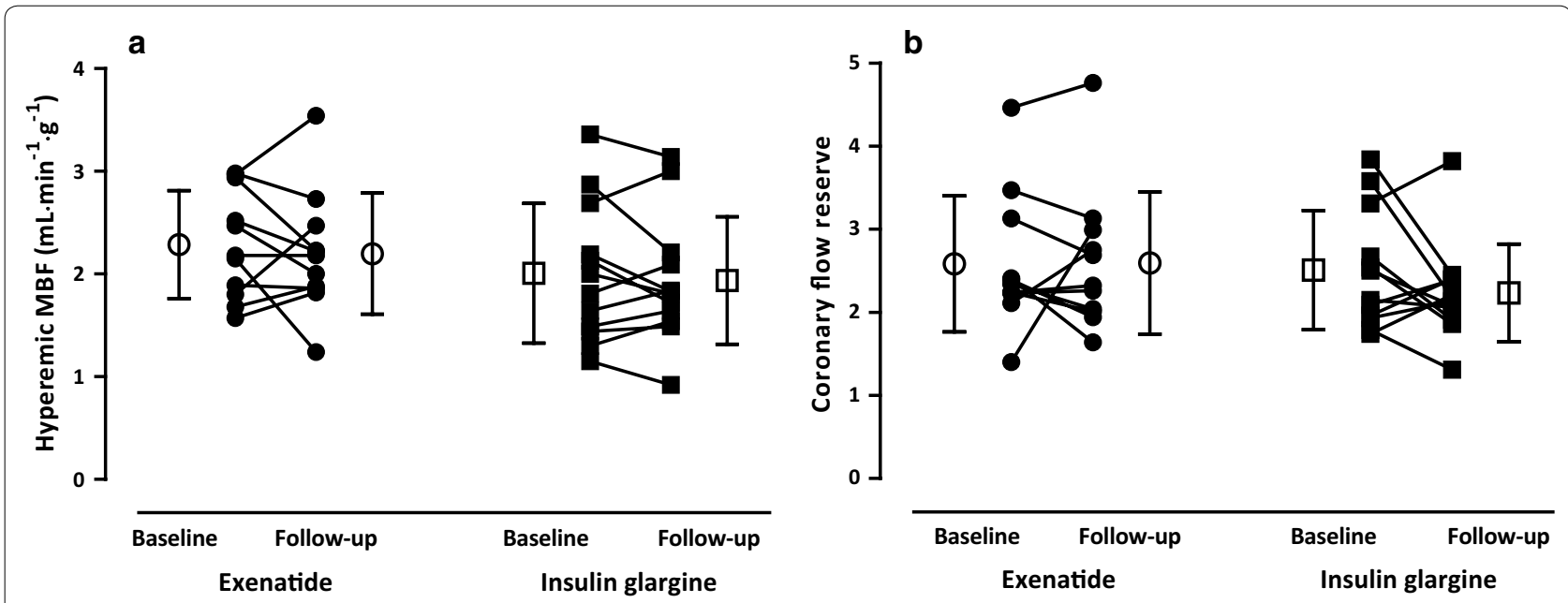

Fig. 5 a Hyperemic myocardial blood flow (MBF) and $\mathbf{b}$ coronary flow reserve in type 2 diabetic patients at baseline and after 26-weeks of exenatide [dots; open dots (mean \pm SD)] versus insulin glargine [squares; open squares (mean \pm SD)] 


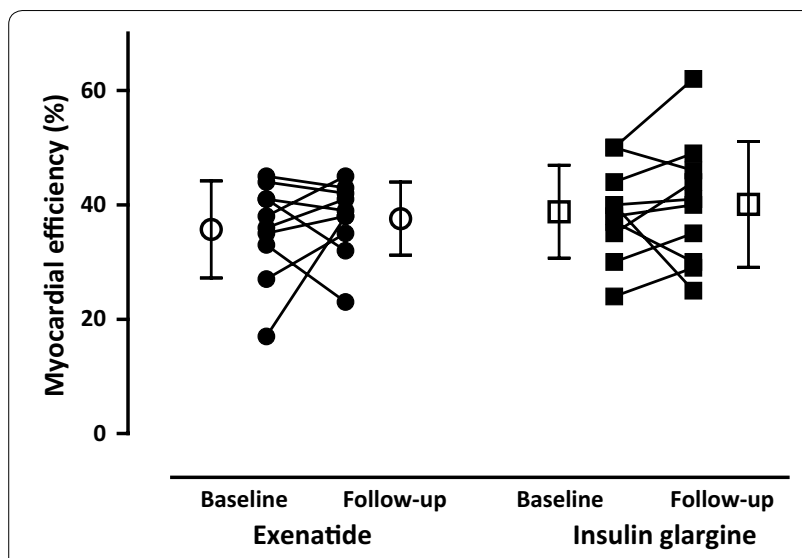

Fig. 6 Myocardial efficiency in type 2 diabetic patients at baseline and after 26-weeks of exenatide [dots; open dots (mean \pm SD)] versus insulin glargine [squares; open squares (mean $\pm \mathrm{SD})$ ]

use, oxygen consumption and myocardial efficiency were seen after albiglutide in non-diabetic patients with heart failure [53]. None of these studies, however, examined the impact on actual oxidative metabolism.

In contrary to the proposed therapeutic strategy in $\mathrm{HF}$, enhancing myocardial glucose metabolism, it has been hypothesized that insulin resistance could protect the heart from substrate overload in the diabetic heart by decreasing the excess energy as myocytes are unable to convert this excess energy into mechanical energy [54]. Agents that can lower serum levels of substrates, thereby reversing the substrate overload, are therefore expected to reverse the metabolic and contractile dysfunction in the diabetic heart [54].

\section{Limitations}

The limited sample size may have obscured potential cardiac effects of exenatide. Furthermore, 2 T2DM patients had severe LV systolic dysfunction. It is indeed well known that diastolic dysfunction frequently occurs in patients with diabetes (and obesity in general). Unfortunately, specific diastolic function parameters were not included in the current study protocol. Besides, CAD may have additionally influenced CFR, as obstructive coronary lesions were not excluded in the present study. However, these effects are likely to be small as this randomized pilot trial did not indicate any significant alterations on the investigated parameters. Furthermore, participants were all male. Therefore, these results cannot be extrapolated to female T2DM patients without further studies. Although, metabolic effects of exenatide are likely to be independent of sex [55]. To evaluate patient's compliance all empty exenatide- and insulin glargine pens were collected. However, serum levels of exenatide were not measured. Furthermore, this study was conducted in patients with mild LV systolic dysfunction. In general, more advanced stages of HF are accompanied by cardiac device therapy prohibiting CMR imaging. Earlier data suggest that myocardial metabolism is particularly abnormal in severe HF, therefore GLP-1RA effects could be more pronounced in those patients.

\section{Conclusions}

In T2DM patients with modest systolic dysfunction, myocardial efficiency was not impaired compared to BMI-matched healthy controls. Furthermore, 26 weeks of exenatide or insulin glargine did not result in changes in cardiac function, MBF, or oxidative metabolism.

\section{Abbreviations \\ BMI: body mass index; BP: blood pressure; CAD: coronary artery disease; CFR: coronary flow reserve; CMR: cardiovascular magnetic resonance imaging; CT: computed tomography; DCE: delayed contrast enhancement; GLP-1: glucagon like peptide-1; GLP-1RA: glucagon like peptide-1 receptor agonist; HbA1c: hemoglobin A1c; HDL: high-density lipoprotein; HF: heart failure; HR: heart rate; $\mathrm{k}_{2}$ : rate constant for tracer washout from myocardial tissue; LDL: low-density lipoprotein; LV: left ventricular; LVEF: left ventricular ejection frac- tion; MBF: myocardial blood flow; $\mathrm{MRI}$ : magnetic resonance imaging; $\mathrm{MVO}_{2}$ : myocardial oxygen consumption; PET: positron emission tomography; RPP: rate pressure product; SBP: systolic blood pressure; SSFP: steady-state free precession; T2DM: type 2 diabetes mellitus.}

\section{Authors' contributions}

WJYC participated in the acquisition of the data, coordination of the study, interpreted the data, performed the statistical analysis and drafted the manuscript. MD contributed to the design of the study, acquisition of the data and interpretation of the data. KDB participated in acquisition of the data, interpretation of the data and critically reviewed the manuscript. $\mathrm{HJH}$ participated in the acquisition of the data, the interpretation of the data and critically reviewed the manuscript. LFHJR contributed to the acquisition of the data, to the interpretation of the data and critically reviewed the manuscript. ACVR contributed to the interpretation of the data and critically reviewed the manuscript. MHHK participated in the interpretation of the data and critically reviewed the manuscript. AAL participated in analysis and interpretation of the data, and critically reviewed the manuscript. PK contributed to the design of the study, participated in the analysis and interpretation of the data, and drafted the manuscript. All authors read and approved the final manuscript.

\section{Author details}

1 Diabetes Center/Department of Internal Medicine, VU University Medical Center, de Boelelaan 1117, 1081 HV Amsterdam, The Netherlands. ${ }^{2}$ Department of Cardiology, VU University Medical Center, de Boelelaan 1117, 1081 HV Amsterdam, The Netherlands. ${ }^{3}$ Department of Radiology and Nuclear Medicine, VU University Medical Center, de Boelelaan 1117, 1081 HV Amsterdam, The Netherlands.

\section{Acknowledgements}

Michaela Diamant, who designed and supervised this research, died on 9 April 2014.

\section{Competing interests}

MD was consultant for Abbott, Astra Zeneca, Bristol-Myers Squibb (BMS), Boehringer Ingelheim, Eli Lilly, Gl Dynamics, Inc., Merck Sharp \& Dohme (MSD), Novo Nordisk, Poxel Pharma and Sanofi, and speaker for BMS/Astra Zeneca, Eli Lilly, Novo Nordisk and Sanofi. MD received research grants from Abbott, BMS-Astra, Boehringer Ingelheim, Eli Lilly, Medtronic, MSD, Novo Nordisk and Sanofi. All above mentioned payments were directly transferred to the Diabetes Center non-profit Research Foundation. Other authors declare no duality of interests. 


\section{Availability of data and materials}

The data that support the findings of this study are available from the corresponding author upon reasonable request.

\section{Ethics approval and consent to participate}

All participants gave written informed consent. The study protocol was approved by the Medical Ethics Review committee of the VU University Medical Center, and was performed in full compliance with the declaration of Helsinki.

\section{Funding}

This investigator-initiated study was supported by Eli Lilly, The Netherlands, which had a partnership with Amylin, the manufacturer of exenatide at the time this trial was designed and data collection was performed.

\section{Publisher's Note}

Springer Nature remains neutral with regard to jurisdictional claims in published maps and institutional affiliations.

Received: 2 March 2017 Accepted: 6 May 2017

Published online: 19 May 2017

\section{References}

1. McMurray JJ, Gerstein HC, Holman RR, Pfeffer MA. Heart failure: a cardiovascular outcome in diabetes that can no longer be ignored. Lancet Diabetes Endocrinol. 2014;2:843-51.

2. Erdmann E, Charbonnel B, Wilcox RG, Skene AM, Massi-Benedetti M, Yates J, Tan M, Spanheimer R, Standl E, Dormandy JA. Pioglitazone use and heart failure in patients with type 2 diabetes and preexisting cardiovascular disease: data from the PROactive study (PROactive 08). Diabetes Care. 2007:30:2773-8.

3. Evans JM, Ogston SA, Emslie-Smith A, Morris AD. Risk of mortality and adverse cardiovascular outcomes in type 2 diabetes: a comparison of patients treated with sulfonylureas and metformin. Diabetologia. 2006:49:930-6.

4. Scirica BM, Bhatt DL, Braunwald E, Steg PG, Davidson J, Hirshberg B, Ohman P, Frederich R, Wiviott SD, Hoffman EB, Cavender MA, Udell JA, Desai NR, Mosenzon O, McGuire DK, Ray KK, Leiter LA, Raz I. Saxagliptin and cardiovascular outcomes in patients with type 2 diabetes mellitus. N Engl J Med. 2013;369:1317-26

5. Zinman B, Wanner C, Lachin JM, Fitchett D, Bluhmki E, Hantel S, Mattheus M, Devins T, Johansen OE, Woerle HJ, Broedl UC, Inzucchi SE. Empagliflozin, cardiovascular outcomes, and mortality in type 2 diabetes. N Engl J Med. 2015:373:2117-28.

6. Marso SP, Daniels GH, Brown-Frandsen K, Kristensen P, Mann JF, Nauck MA, Nissen SE, Pocock S, Poulter NR, Ravn LS, Steinberg WM, Tockner M, Zinman B, Bergenstal RM, Buse JB. Liraglutide and cardiovascular outcomes in type 2 diabetes. N Engl J Med. 2016;375:311-22.

7. Sokos GG, Nikolaidis LA, Mankad S, Elahi D, Shannon RP. Glucagonlike peptide-1 infusion improves left ventricular ejection fraction and functional status in patients with chronic heart failure. J Cardiac Fail. 2006;12:694-9.

8. Thrainsdottir I, Malmberg K, Olsson A, Gutniak M, Ryden L. Initial experience with GLP-1 treatment on metabolic control and myocardial function in patients with type 2 diabetes mellitus and heart failure. Diab Vasc Dis Res. 2004;1:40-3.

9. Nystrom T, Gutniak MK, Zhang Q, Zhang F, Holst JJ, Ahren B, Sjoholm A. Effects of glucagon-like peptide-1 on endothelial function in type 2 diabetes patients with stable coronary artery disease. Am J Physiol Endocrinol Metab. 2004;287:E1209-15.

10. Koska J, Sands M, Burciu C, D'Souza KM, Raravikar K, Liu J, Truran S, Franco DA, Schwartz EA, Schwenke DC, D'Alessio D, Migrino RQ, Reaven PD. Exenatide protects against glucose- and lipid-induced endothelial dysfunction: evidence for direct vasodilation effect of GLP-1 receptor agonists in humans. Diabetes. 2015;64:2624-35.

11. Ashrafian H, Frenneaux MP, Opie LH. Metabolic mechanisms in heart failure. Circulation. 2007;116:434-48.
12. Doehner W, Frenneaux M, Anker SD. Metabolic impairment in heart failure: the myocardial and systemic perspective. J Am Coll Cardiol. 2014:64:1388-400.

13. Marti CN, Gheorghiade M, Kalogeropoulos AP, Georgiopoulou W, Quyyumi AA, Butler J. Endothelial dysfunction, arterial stiffness, and heart failure. J Am Coll Cardiol. 2012;60:1455-69.

14. Yki-Jarvinen $\mathrm{H}$, Juurinen $\mathrm{L}$, Alvarsson M, Bystedt T, Caldwell I, Davies M Lahdenpera S, Nijpels G, Vahatalo M. Initiate Insulin by Aggressive Titration and Education (INITIATE): a randomized study to compare initiation of insulin combination therapy in type 2 diabetic patients individually and in groups. Diabetes Care. 2007:30:1364-9.

15. Beek AM, van Rossum AC. Use of cardiovascular magnetic resonance imaging in the assessment of left ventricular function, scar and viability in patients with ischaemic cardiomyopathy and chronic myocardial infarction. Heart. 2010;96:1494-501.

16. Cerqueira MD, Weissman NJ, Dilsizian V, Jacobs AK, Kaul S, Laskey WK, Pennell DJ, Rumberger JA, Ryan T, Verani MS. Standardized myocardial segmentation and nomenclature for tomographic imaging of the heart: a statement for healthcare professionals from the Cardiac Imaging Committee of the Council on Clinical Cardiology of the American Heart Association. Circulation. 2002;105:539-42.

17. Sun KT, Chen K, Huang SC, Buxton DB, Hansen HW, Kim AS, Siegel S, Choi Y, Muller P, Phelps ME, Schelbert HR. Compartment model for measuring myocardial oxygen consumption using [1-11C]acetate. J Nucl Med. 1997;38:459-66.

18. Timmer SA, Lubberink M, van Rossum AC, Lammertsma AA, Knaapen P. Reappraisal of a single-tissue compartment model for estimation of myocardial oxygen consumption by [11C]acetate PET: an alternative to conventional monoexponential curve fitting. Nucl Med Commun. 2011;32:59-62

19. Harms HJ, Knaapen $P$, de HS, Halbmeijer R, Lammertsma AA, Lubberink M. Automatic generation of absolute myocardial blood flow images using [15O]H2O and a clinical PET/CT scanner. Eur J Nucl Med Mol Imaging. 2011;38:930-9.

20. Knaapen P, Germans T, Knuuti J, Paulus WJ, Dijkmans PA, Allaart CP, Lammertsma AA, Visser FC. Myocardial energetics and efficiency: current status of the noninvasive approach. Circulation. 2007;115:918-27.

21. Sun KT, Yeatman LA, Buxton DB, Chen K, Johnson JA, Huang SC, Kofoed KF, Weismueller S, Czernin J, Phelps ME, Schelbert HR. Simultaneous measurement of myocardial oxygen consumption and blood flow using [1-carbon-11]acetate. J Nucl Med. 1998;39:272-80

22. Porenta G, Cherry S, Czernin J, Brunken R, Kuhle W, Hashimoto T, Schelbert HR. Noninvasive determination of myocardial blood flow, oxygen consumption and efficiency in normal humans by carbon-11 acetate positron emission tomography imaging. Eur J Nucl Med. 1999;26:1465-74.

23. Vinik Al, Maser RE, Mitchell BD, Freeman R. Diabetic autonomic neuropathy. Diabetes Care. 2003;26:1553-79.

24. Ferrannini E, Cushman WC. Diabetes and hypertension: the bad companions. Lancet. 2012:380:601-10.

25. Di Carli MF, Bianco-Batlles D, Landa ME, Kazmers A, Groehn H, Muzik O, Grunberger G. Effects of autonomic neuropathy on coronary blood flow in patients with diabetes mellitus. Circulation. 1999;100:813-9.

26. Rijzewijk LJ, van der Meer RW, Lamb HJ, de Jong HW, Lubberink M, Romijn JA, Bax JJ, de RA, Twisk JW, Heine RJ, Lammertsma AA, Smit JW, Diamant M. Altered myocardial substrate metabolism and decreased diastolic function in nonischemic human diabetic cardiomyopathy: studies with cardiac positron emission tomography and magnetic resonance imaging. J Am Coll Cardiol. 2009;54:1524-32.

27. Iozzo P, Chareonthaitawee P, Rimoldi O, Betteridge DJ, Camici PG, Ferrannini E. Mismatch between insulin-mediated glucose uptake and blood flow in the heart of patients with Type II diabetes. Diabetologia. 2002:45:1404-9.

28. Peterson LR, Saeed IM, McGill JB, Herrero P, Schechtman KB, Gunawardena R, Recklein CL, Coggan AR, DeMoss AJ, Dence CS, Gropler RJ. Sex and type 2 diabetes: obesity-independent effects on left ventricula substrate metabolism and relaxation in humans. Obesity (Silver Spring). 2012;20:802-10.

29. Alexanderson E, Rodriguez-Valero M, Martinez A, Calleja R, Lamothe PA Sierra C, Garcia-Rojas L, Talayero JA, Cruz P, Meave A, Alexanderson G. Endothelial dysfunction in recently diagnosed type 2 diabetic patients evaluated by PET. Mol Imaging Biol. 2009;11:1-5. 
30. Rimoldi OE, Drake-Holland AJ, Noble MI, Camici PG. Basal and hyperaemic myocardial blood flow in regionally denervated canine hearts: an in vivo study with positron emission tomography. Eur J Nucl Med Mol Imaging. 2007;34:197-205.

31. Stanley WC, Recchia FA, Lopaschuk GD. Myocardial substrate metabolism in the normal and failing heart. Physiol Rev. 2005;85:1093-129.

32. Ouwens DM, Diamant M. Myocardial insulin action and the contribution of insulin resistance to the pathogenesis of diabetic cardiomyopathy. Arch Physiol Biochem. 2007;113:76-86.

33. Tuunanen $\mathrm{H}$, Ukkonen $\mathrm{H}$, Knuuti J. Myocardial fatty acid metabolism and cardiac performance in heart failure. Curr Cardiol Rep. 2008;10:142-8.

34. Iozzo P. Metabolic toxicity of the heart: insights from molecular imaging. Nutr Metab Cardiovasc Dis. 2010;20:147-56.

35. Hattori N, Tamaki N, Kudoh T, Masuda I, Magata Y, Kitano H, Inubushi M, Tadamura E, Nakao K, Konishi J. Abnormality of myocardial oxidative metabolism in noninsulin-dependent diabetes mellitus. J Nucl Med 1998;39:1835-40.

36. Tuunanen $\mathrm{H}$, Engblom E, Naum A, Scheinin M, Nagren K, Airaksinen J, Nuutila P, lozzo P, Ukkonen H, Knuuti J. Decreased myocardial free fatty acid uptake in patients with idiopathic dilated cardiomyopathy: evidence of relationship with insulin resistance and left ventricular dysfunction. J Card Fail. 2006:12:644-52

37. Nichols AB, Pearson MH, Sciacca RR, Cannon PJ. Left ventricular mechanical efficiency in coronary artery disease. J Am Coll Cardiol. 1986;7:270-9.

38. Robinson LE, Holt TA, Rees K, Randeva HS, O'Hare JP. Effects of exenatide and liraglutide on heart rate, blood pressure and body weight: systematic review and meta-analysis. BMJ Open. 2013;3:e001986. doi:10.1136/ bmjopen-2012-001986.

39. Blonde L, Pencek R, MacConell L. Association among weight change, glycemic control, and markers of cardiovascular risk with exenatide once weekly: a pooled analysis of patients with type 2 diabetes. Cardiovasc Diabetol. 2015;14:12.

40. Simo R, Guerci B, Schernthaner G, Gallwitz B, Rosas-Guzman J, Dotta F, Festa A, Zhou M, Kiljanski J. Long-term changes in cardiovascular risk markers during administration of exenatide twice daily or glimepiride: results from the European exenatide study. Cardiovasc Diabetol. 2015;14:116.

41. Wysham CH, MacConell LA, Maggs DG, Zhou M, Griffin PS, Trautmann ME. Five-year efficacy and safety data of exenatide once weekly: long-term results from the DURATION-1 randomized clinical trial. Mayo Clin Proc. 2015;90:356-65.

42. Nathanson D, Ullman B, Lofstrom U, Hedman A, Frick M, Sjoholm A Nystrom T. Effects of intravenous exenatide in type 2 diabetic patients with congestive heart failure: a double-blind, randomised controlled clinical trial of efficacy and safety. Diabetologia. 2012;55:926-35.

43. Halbirk M, Norrelund H, Moller N, Holst JJ, Schmitz O, Nielsen R, NielsenKudsk JE, Nielsen SS, Nielsen TT, Eiskjaer H, Botker HE, Wiggers H. Cardio vascular and metabolic effects of 48-h glucagon-like peptide-1 infusion in compensated chronic patients with heart failure. Am J Physiol Heart Circ Physiol. 2010;298:H1096-102.

44. Jorsal A, Kistorp C, Holmager P, Tougaard RS, Nielsen R, Hanselmann A, Nilsson B, Moller JE, Hjort J, Rasmussen J, Boesgaard TW, Schou M, Videbaek L, Gustafsson I, Flyvbjerg A, Wiggers H, Tarnow L. Effect of liraglutide, a glucagon-like peptide-1 analogue, on left ventricular function in stable chronic heart failure patients with and without diabetes (LIVE)-a multicentre, double-blind, randomised, placebo-controlled trial. Eur J Heart Fail. 2017;19:67-77.
45. Margulies KB, Hernandez AF, Redfield MM, Givertz MM, Oliveira GH, Cole R, Mann DL, Whellan DJ, Kiernan MS, Felker GM, McNulty SE, Anstrom KJ, Shah MR, Braunwald E, Cappola TP. Effects of liraglutide on clinical stability among patients with advanced heart failure and reduced ejection fraction: a randomized clinical trial. JAMA. 2016:316:500-8.

46. Gejl M, Sondergaard HM, Stecher C, Bibby BM, Moller N, Botker HE, Hansen SB, Gjedde A, Rungby J, Brock B. Exenatide alters myocardial glucose transport and uptake depending on insulin resistance and increases myocardial blood flow in patients with type 2 diabetes. J Clin Endocrinol Metab. 2012;97:E1165-9.

47. Faber R, Zander M, Pena A, Michelsen MM, Mygind ND, Prescott E. Effect of the glucagon-like peptide-1 analogue liraglutide on coronary microvascular function in patients with type 2 diabetes-a randomized, single-blinded, cross-over pilot study. Cardiovasc Diabetol. 2015;14:41.

48. Gurkan E, Tarkun I, Sahin T, Cetinarslan B, Canturk Z. Evaluation of exenatide versus insulin glargine for the impact on endothelial functions and cardiovascular risk markers. Diabetes Res Clin Pract. 2014;106:567-75.

49. Torimoto K, Okada Y, Mori H, Otsuka T, Kawaguchi M, Matsuda M, Kuno F, Sugai K, Sonoda S, Hajime M, Tanaka K, Arao T, Tanaka Y. Effects of exenatide on postprandial vascular endothelial dysfunction in type 2 diabetes mellitus. Cardiovasc Diabetol. 2015;14:25.

50. Nikolaidis LA, Elahi D, Hentosz T, Doverspike A, Huerbin R, Zourelias L, Stolarski C, Shen YT, Shannon RP. Recombinant glucagon-like peptide-1 increases myocardial glucose uptake and improves left ventricular performance in conscious dogs with pacing-induced dilated cardiomyopathy. Circulation. 2004;110:955-61.

51. Gejl M, Lerche S, Mengel A, Moller N, Bibby BM, Smidt K, Brock B, Sondergaard H, Botker HE, Gjedde A, Holst JJ, Hansen SB, Rungby J. Influence of GLP-1 on myocardial glucose metabolism in healthy men during normoor hypoglycemia. PLOS ONE. 2014;9:e83758.

52. McCormick LM, Hoole SP, White PA, Read PA, Axell RG, Clarke SJ, O'Sullivan $M$, West NE, Dutka DP. Pre-treatment with glucagon-like Peptide-1 protects against ischemic left ventricular dysfunction and stunning without a detected difference in myocardial substrate utilization. JACC Cardiovasc Interv. 2015;8:292-301.

53. Lepore JJ, Olson E, Demopoulos L, Haws T, Fang Z, Barbour AM, Fossler M, Davila-Roman VG, Russell SD, Gropler RJ. Effects of the novel long-acting GLP-1 agonist, albiglutide, on cardiac function, cardiac metabolism, and exercise capacity in patients with chronic heart failure and reduced ejection fraction. JACC Heart Fail. 2016:4:559-66.

54. Taegtmeyer H, Beauloye C, Harmancey R, Hue L. Insulin resistance protects the heart from fuel overload in dysregulated metabolic states. Am J Physiol Heart Circ Physiol. 2013;305:H1693-7.

55. Pencek R, Blickensderfer A, Li Y, Brunell SC, Anderson PW. Exenatide twice daily: analysis of effectiveness and safety data stratified by age, sex, race, duration of diabetes, and body mass index. Postgrad Med. $2012 ; 124: 21-32$.

\section{Submit your next manuscript to BioMed Central and we will help you at every step:}

- We accept pre-submission inquiries

- Our selector tool helps you to find the most relevant journal

- We provide round the clock customer support

- Convenient online submission

- Thorough peer review

- Inclusion in PubMed and all major indexing services

- Maximum visibility for your research

Submit your manuscript at www.biomedcentral.com/submit
BioMed Central 